\title{
Fitosociologia de malezas asociadas a cultivos de zanahoria en dos municipios de Boyacá
}

\author{
Phytosociology of weeds associated with carrot crops \\ in two municipalities of Boyacá
}

\author{
Luís David Córdoba-Patiño' \\ Elberth Hernando Pinzon-Sandoval ${ }^{2}$
}

DOI: https://doi.org/10.19053/01228420.v18.n3.2021.13752

RESUMEN: Con la finalidad de conocer la fitosociología de las malezas asociadas a un cultivo de zanahoria (Daucus carota L.) bajo condiciones de los municipios de Ventaquemada y JenesanoBoyacá, se seleccionó un lote por municipio destinado al cultivo de zanahoria y se realizó un trazado en forma de W cubriendo un área de $500 \mathrm{~m}^{2}$. Se calculó la densidad relativa, frecuencia relativa, dominancia relativa y el índice de valor de importancia (IVI), así como los índices de diversidad Alpha y Beta para las zonas muestreadas. Se contabilizó un total de 6 familias y 11 especies, de estas el 63,64\% estuvo representado por plantas anuales y $36,36 \%$ por plantas perennes. La clase Liliopsida (Monocotiledónea) fue representada por la familia Poaceae. Mientras que la clase Magnoliopsida (Dicotiledónea), fue representada por las familias: Asteraceae, Brassicaceae, Boraginaceae, Leguminosaceae, Polygonaceae siendo la ultima la que aporto el mayor número de especies. La especie $R$. crispus y $P$. nepalense fueron las que presentaron los mayores valores de índice de valor de Importancia (IVI) con 0,953 y 0,959 , respectivamente. De acuerdo, con los índices de diversidad de Shannon-Wiener y dominancia de Simpson las zonas evaluadas presentaron una baja diversidad de especies y una alta probabilidad especies dominantes. Los resultados encontrados pueden servir de base y herramienta a los productores de zanahoria de las zonas evaluadas, para definir los planes de manejo de las malezas asociadas y así optimizar los rendimientos en este cultivo.

PALABRAS CLAVE: Competencia; Diversidad; Dominancia; Daucus carota; Polygonum nepalense; Rumex crispus.
ABSTRACT: In order to know the phytosociology of the weeds associated with a carrot crop (Daucus carota L.) under conditions of the municipalities of Ventaquemada and Jenesano-Boyacá, a lot was selected per municipality for carrot cultivation and a plot was made W-shaped covering an area of $500 \mathrm{~m}^{2}$. Relative density, relative frequency, relative dominance and the importance value index (IVI) were calculated, as well as the Alpha and Beta diversity indices for the sampled areas. A total of 6 families and 11 species were counted, of these $63.64 \%$ were represented by annual plants and $36.36 \%$ by perennial plants. The class Liliopsida (Monocotyledonous) was represented by the family Poaceae. While the Magnoliopsida (Dicotyledonous) class was represented by the families: Asteraceae, Brassicaceae, Boraginaceae, Leguminosaceae, Polygonaceae, the latter being the one that contributed the largest number of species. The species $R$. crispus and $P$. nepalense were those that presented the highest values of Importance value index (IVI) with 0.953 and 0.959 , respectively. Accordingly, with the Shannon-Wiener diversity index and Simpson dominance, the evaluated areas presented a low species diversity and a high probability of dominant species. The results found can serve as a basis and tool for the carrot producers in the evaluated areas, to define the management plans of the associated weeds and thus optimize the yields in this crop.

KEY WORDS: Competence; Diversity; Dominance; Daucus Carota; Polygonum nepalense, Rumex crispus. 


\section{Introducción}

La zanahoria (Daucus carota L.), es el miembro más cultivado de la familia Apiaceae (Poleshi et al., 2018), así como también la principal hortaliza del grupo de las raíces tuberosas (Resende et al., 2016; Patkowska, 2021). es una especie originaria de zonas templadas, pero se cultiva en regiones tropicales y subtropicales (Cardenal et al., 2016), se encuentra entre los diez cultivos de hortalizas con mayor importancia económica del mundo en términos de área y valor del mercado (Schmid et al., 2021). En Colombia, el cultivo de zanahoria alcanzó un área sembrada de 10.933 hectáreas para el año 2019, siendo Boyacá el segundo departamento con mayor producción y con un aporte de 29,1\% a la producción total nacional, superado únicamente por el departamento de Cundinamarca (Agronet, 2020).

En el sistema productivo de zanahoria, el manejo apropiado de la competencia interespecífica constituye un factor determinante en el rendimiento y éxito comercial del cultivo(Dotor et al., 2018). En cultivos hortícolas el control de malezas constituye una de las labores más difíciles y costosas (Vera \& Enciso, 2017). Se indica que la capacidad competitiva de la planta de zanahoria es baja debido a que la emergencia y el crecimiento temprano son relativamente lentos (Reginaldo et al., 2021; Šuk et al., 2021).

De acuerdo con Coelho et al. (2009) al no generar un control rápido de las plantas malezas en este cultivo, se puede causar una pérdida de rendimiento de hasta un 94\%. Esto como consecuencia de la interferencia causada por las mismas (Pitelli, 1987). El grado de interferencia de las malezas en los cultivos agrícolas se puede definir como la reducción porcentual del rendimiento del cultivo causada por la convivencia con la comunidad de malezas (Kuva et al., 2007). Este grado de interferencia depende de factores relacionados con el cultivo en sí (especie o variedad, espaciamiento y densidad de siembra), la comunidad de malezas (composición específica, densidad y distribución) y el tiempo y duración del período de coexistencia (Biffe et al., 2018).

El conocimiento de la estructura de una comunidad de malezas es muy importante. Debido a que, para generar un adecuado programa de manejo es necesario establecer un orden de prioridades entre las especies presentes(Kuva et al., 2007). Esto debido a que existen especies dominantes, que originan la mayor parte de la interferencia, especies secundarias, presentes en menor densidad y cobertura, y especies acompañantes, cuya presencia es ocasional y que difícilmente generan problemas económicos a los cultivos (Kuva et al., 2007).

En un agroecosistema, las malezas causan interferencia en diferentes grados, dependiendo de sus características individuales; las diferentes condiciones del suelo y del clima en las diferentes regiones agroecológicas influyen en la composición florística y el predominio de una especie de maleza sobre otra, adicionalmente para programar prácticas que conlleven al manejo adecuado 
de malezas en los diversos cultivos es indispensable identificar si la composición de las comunidades de malezas y sus características cualitativas y cuantitativas difieren de una región agroecológica a otra (Cabrera et al., 2019).

Carvalho \& Guzzo (2008) destacan la importancia de conocer la composición de la comunidad de malezas como un factor fundamental para determinar el grado de interferencia, esto debido a que las poblaciones que componen la comunidad tienen una fuerte relación entre sí, esto se puede llegar a estudiar mediante índices fitosociológicos; entre estos, el que mejor expresa esta relación es el índice de importancia relativa (Carvalho et al., 2008).

Uno de los métodos o herramientas más utilizados para analizar las comunidades de malezas presentes en un cultivo es el estudio fitosociológico, el cual permite analizar las comunidades de malezas a partir de la composición y estructura, permitiendo de esta forma tener una visión global de la composición, estructura y distribución de las especies de plantas en una comunidad determinada (Concenço et al., 2017; Quintero-Pertuz et al., 2021). Para esto, es necesario identificar las distintas especies integrantes de la comunidad, obtener datos de abundancia, densidad, frecuencia de aparición, entre otros caracteres cuantitativos, mediante inventarios florísticos, a partir de los cuales, se estiman índices que permiten caracterizar la vegetación (Booth et al., 2003).

El objetivo de esta investigación es evaluar las poblaciones de malezas asociadas al cultivo de zanahoria en dos municipios del departamento de Boyacá, con el fin de generar herramientas técnicas para la toma de decisiones frente al esquema de manejo de malezas para el sistema productivo de la zanahoria bajo las condiciones de estudio.

\section{Materiales y Métodos}

\section{Localización}

Se seleccionaron dos lotes dedicados a la producción de zanahoria (Daucus carota L.) con una extensión aproximada de una hectárea cada uno, pertenecientes a los municipios de Ventaquemada ubicado a $2630 \mathrm{msnm}$, temperatura promedio de $16^{\circ} \mathrm{C}$ y coordenadas N 5'21'25.0" W $73^{\circ} 32^{\prime} 14.1^{\prime \prime}$ y el municipio de Jenesano ubicado a $2076 \mathrm{msnm}$, temperatura promedio de $18^{\circ} \mathrm{C}$ y coordenadas N 5'24'11.5290" W 7325'8.0100".

\section{Metodología}

Para caracterizar las especies de malezas asociadas se tomaron muestras en cada lote seleccionado por municipio, se realizó un trazado en forma de W cubriendo un área de $500 \mathrm{~m}^{2}$ siguiendo la metodología descrita por Cardenal et al. (2016). Se empleó un marco en pvc de 0,25 cm x 0,25 cm de acuerdo 
con Prates et al. (2019), con el fin de tomar 9 muestras por lote con una profundidad de $30 \mathrm{~cm}$, una vez homogenizadas las muestras estas se dispusieron en 9 bandejas de aluminio de $31 \mathrm{~cm}$ de largo, por $25 \mathrm{~cm}$ de ancho y $5 \mathrm{~cm}$ de profundidad. Se mantuvieron con adecuadas condiciones de agua, luz y humedad.

La identificación de las especies inicio 20 días después de la disposición de las muestras, una vez las especies iniciaron a emerger se realizó la respectiva identificación a nivel de plántula, Para el reconocimiento e identificación de las especies se utilizaron los manuales de reconocimiento de plantas para zonas de clima frio (Fuentes et al., 2011; Gámez et al., 2018). El experimento tuvo una duración de 100 días, con cinco conteos de malezas cada 20 días, hasta que no se evidencio más germinación de plántula en las bandejas.

\section{Análisis de datos}

Para determinar el índice de valor de importancia se empleó la siguiente formula (Gámez et al., 2012):

$$
\mathrm{IVI}=\mathrm{Fr}+\mathrm{Dr}+\mathrm{dr}
$$

Siendo:

$$
\text { Frecuencia Relativa }(\mathrm{Fr})=\frac{\text { Frecuencia absoluta por especie }}{\text { Frecuencia total de todas las especies }}
$$

Densidad relativa $(\mathrm{Dr})=\frac{\text { Densidad absoluta por especie }}{\text { Densidad total de todas las especies }}$

Dominancia relativa $(\mathrm{dr})=\frac{\text { Dominancia por especie }}{\text { Dominancia total }}$

\begin{tabular}{|c|c|c|}
\hline Índice & Ecuación & Parámetros \\
\hline Índice Dominancia Simpson (D) & $\mathrm{D}=\Sigma\left(\frac{n i}{N}\right) 2$ & \multirow{2}{*}{$\begin{aligned} \mathrm{ni}= & \text { número de individuos de las especies } \\
\mathrm{N}= & \text { número total de individuos de todas } \\
& \text { las especies } \\
\mathrm{Pi}= & \text { abundancia proporcional de una } \\
& \text { especie dada }\end{aligned}$} \\
\hline Índice Shannon-Wiener ( $\mathrm{H}^{\prime}$ ) & $\mathrm{H}^{\prime}=-\Sigma[$ pi (In pi ) $]$ & \\
\hline
\end{tabular}

Se determinaron los índices de diversidad Alfa $(\alpha)$ :

\begin{tabular}{|c|c|c|}
\hline Índice & Ecuación & Parámetros \\
\hline Índice Jaccard $\left(\mathrm{S}_{\mathrm{J}}\right)$ & $S_{\mathrm{J}}=\mathrm{j} /(a+b+j)$ & \multirow{2}{*}{$\begin{aligned} \mathrm{j}= & \text { número de especies encontradas en las } \\
& \text { dos comunidades } \\
\mathrm{a}= & \text { número de especies encontradas solo en } \\
& \text { una comunidad } \mathrm{a} \\
\mathrm{b}= & \text { número de especies encontradas solo en } \\
& \text { una comunidad } \mathrm{b}\end{aligned}$} \\
\hline Índice Sorensen (S Índice) & $S_{S}=2 j /(a+b+2 j)$ & \\
\hline
\end{tabular}

Se determinaron los índices de diversidad Beta ( $\beta)$ : 
Los cálculos de los anteriores índices y parámetros se realizaron a través del programa informático Microsoft ${ }^{\circledR}$ Office Excel 2013 y el software Past versión 3.17.

\section{Resultados y Discusión}

Se determinó un total de 11 especies pertenecientes a 6 familias, de las cuales, en lo que respecta a su ciclo de vida, el 63,64\% estuvo representado por plantas anuales y 36,36,79\% por plantas perennes (Tabla I). Gámez et al. (2012) indican que el predominio de las plantas anuales sobre las perennes puede ser la respuesta al uso excesivo del control químico.

TABLA I. Clasificación de las especies asociadas al cultivo de zanahoria en lotes productores de los municipios de Ventaquemada y Jenesano. Ciclo A: Anual, P: Perenne.

\begin{tabular}{|c|c|c|c|c|c|c|}
\hline Clase & Familia & Especie & Nombre común & Ciclo & Ventaquemada & Jenesano \\
\hline \multirow{2}{*}{$\begin{array}{l}\text { Liliopsida } \\
\text { (Monocotiledónea) }\end{array}$} & \multirow{2}{*}{ Poaceae } & Bromus catarthicus Vahl. & Cebadilla & $P$ & $x$ & $x$ \\
\hline & & Lolium temulentum L. & Cizaña, Ray grass & A & & $x$ \\
\hline \multirow{9}{*}{$\begin{array}{l}\text { Magnoliopsida } \\
\text { (Dicotiledónea) }\end{array}$} & \multirow{3}{*}{ Polygonaceae } & Rumex acetosella $\mathrm{L}$. & Envidia & $P$ & $x$ & \\
\hline & & Rumex crispus L. & Lengua de vaca & $P$ & $x$ & $x$ \\
\hline & & Polygonum nepalense Meisn. & Corazón herido & A & $x$ & $x$ \\
\hline & \multirow{2}{*}{ Brassicaceae } & Lepidium bipinnatifidum Desv. & Mastuerzo & A & $x$ & $x$ \\
\hline & & Raphanus raphanistrum L. & Nabo forrajero & A & $x$ & $x$ \\
\hline & Leguminosaceae & Trifolium repens $\mathrm{L}$. & Trébol & $P$ & $x$ & $x$ \\
\hline & \multirow{2}{*}{ Asteraceae } & Senecio vulgaris $\mathrm{L}$. & Yuyito, Senecio & A & $x$ & $x$ \\
\hline & & Sonchus oleoraceus L. & Cerraja, Lechosa & A & $x$ & $x$ \\
\hline & Boraginaceae & Boreqo officinalis $\mathrm{L}$. & Borraja & A & & $x$ \\
\hline
\end{tabular}

La clase monocotiledónea (Liliopsida) fue representada por la familia Poaceae, con dos especies. Por su parte la clase dicotiledónea (Magnoliopsida) estuvo representada por las familias: Asteraceae, Brassicaceae, Boraginaceae, Leguminosaceae, Polygonaceae siendo la ultima la que aporto el mayor número de especies (Tabla I).

En el estudio se encontró la presencia de la familia Poaceae la cual es mencionada en diferentes estudios (Cardenal et al., 2016; Quintero-Pertuz et al., 2021) esto corrobora lo descrito para esta familia botánica considerada como una de las más numerosas, ya que se calcula que esta ocupa un $20 \%$ de la superficie vegetal del mundo (López et al., 2012). De acuerdo, con Bastidas \& López, (1972) reportan a Polygonum segetum, Polygonum nepalense, Rumex 
acetosella, Pennisetum clandestinum como las especies más frecuentes en sistemas productivos de hortalizas. Por su parte, Banda et al. (2012) indican que familias como Poaceae, Fumariaceae, Chenopodiaceae, Polygonaceae, Amaranthaceae Malvaceae, agrupan algunas de las especies de malezas más limitantes. En sistemas de producción de hortalizas como zanahoria y papa en el departamento de Boyacá, las especies de malezas con mayor frecuencia pertenecen a las familias Asteraceae, Polygonaceae y Poaceae (Plaza \& Pedraza, 2007).

Los parámetros poblacionales de las 8 especies de malezas asociadas al cultivo de zanahoria en el municipio de Ventaquemada se presentan en la Tabla II. La especie $R$. crispus fue la de mayor IVI con 0,953, seguida de $P$. nepalense con 0,489 y $R$. acetocella con $0,049 \%$. En cuanto a la frecuencia de aparición la especie $R$. crispus obtuvo el $45,3 \%$; seguida de $P$. nepalense con $25,7 \%$, las demás especies presentaron frecuencias menores al $6 \%$.

TABLA II. Parámetros poblacionales de las especies de malezas encontradas en un lote dedicado a la producción de zanahoria en el municipio de Ventaquemada. ni: Frecuencia absoluta; fr: Frecuencia relativa; Dr: Densidad relativa; dr: Dominancia relativa; IVI: Índice de valor de importancia (IVI).

\begin{tabular}{|c|c|c|c|c|c|}
\hline Especie & ni & fr & Dr & dr & IVI \\
\hline Rumex crispus L. & 295 & 0,453 & 0,295 & 0,2053 & 0,953 \\
\hline Polygonum nepalense Meisn. & 167 & 0,257 & 0,167 & 0,0658 & 0,489 \\
\hline Raphanus raphanistrum L. & 19 & 0,029 & 0,019 & 0,0009 & 0,049 \\
\hline Rumex acetosella L. & 38 & 0,058 & 0,038 & 0,0034 & 0,100 \\
\hline Bromus catarthicus Vahl. & 23 & 0,035 & 0,023 & 0,0012 & 0,060 \\
\hline Trifolium repens $\mathrm{L}$. & 37 & 0,057 & 0,037 & 0,0032 & 0,097 \\
\hline Senecio vulgaris $\mathrm{L}$. & 37 & 0,057 & 0,037 & 0,0032 & 0,097 \\
\hline Sonchus oleoraceus L. & 35 & 0,054 & 0,035 & 0,0029 & 0,092 \\
\hline Número total de individuos (N) & 651 & & & & \\
\hline Número total de especies (S) & 8 & & & & \\
\hline
\end{tabular}

Para el municipio de Jenesano de las 12 especies de malezas asociadas al cultivo de zanahoria, la especie $P$. nepalense fue la de mayor IVI con 0,959, seguida de $B$. catarthicus con 0,342 y L. bipinnatifidum con 0,175. En cuanto a la frecuencia de aparición la especie $P$. nepalense obtuvo el 44,9\%; seguida de $B$. catarthicus con $18,3 \%$ y L. bipinnatifidum con $9,8 \%$, las demás especies presentaron frecuencias menores al 6\% (Tabla III). 
TABLA III. Parámetros poblacionales de las especies de malezas encontradas en un lote destinado a la producción de zanahoria en el municipio de Jenesano. ni: Frecuencia absoluta; fr: Frecuencia relativa; Dr: Densidad relativa; dr: Dominancia relativa; IVI: Índice de valor de importancia (IVI).

\begin{tabular}{|c|c|c|c|c|c|}
\hline Especie & ni & $\mathrm{fr}$ & Dr & dr & IVI \\
\hline Polygonum nepalense Meisn. & 307 & 0,449 & 0,202 & 0,307 & 0,959 \\
\hline Bromus catarthicus Vahl. & 125 & 0,183 & 0,033 & 0,125 & 0,342 \\
\hline Lolium termulentum L. & 32 & 0,047 & 0,002 & 0,032 & 0,081 \\
\hline Raphanus raphanistrum L. & 28 & 0,041 & 0,002 & 0,028 & 0,071 \\
\hline Trifolium repens $\mathrm{L}$. & 23 & 0,034 & 0,001 & 0,023 & 0,058 \\
\hline Lepidium bipinnatifidum Donn. Sm. & 67 & 0,098 & 0,010 & 0,067 & 0,175 \\
\hline Senecio vulgaris $\mathrm{L}$. & 29 & 0,042 & 0,002 & 0,029 & 0,073 \\
\hline Sonchus oleoraceus & 35 & 0,051 & 0,003 & 0,035 & 0,089 \\
\hline Rumex acetosella L. & 21 & 0,031 & 0,001 & 0,021 & 0,053 \\
\hline Boreqo officinalis $\mathrm{L}$. & 16 & 0,023 & 0,001 & 0,016 & 0,040 \\
\hline Número total de individuos (N) & 683 & & & & \\
\hline Número total de especies (S) & 10 & & & & \\
\hline
\end{tabular}

Al analizar de forma general el área bajo estudio la dinámica de las especies malezas asociadas al cultivo de zanahoria se puede indicar que, de acuerdo con el IVI, las especies más importantes son: $P$. nepalense, $R$. crispus, $B$. catarthicus y L. bipinnatifidum debido a que estas se destacan ampliamente frente a las demás especies. Así, las especies con menor IVI fueron: R. raphanistrum y $B$. officinalis.

De acuerdo, con Plaza \& Pedraza, (2007), la alta frecuencia y el incremento poblacional de las especies $P$. nepalense y $R$. crispus, pertenecientes a la familia Polygonaceae están dados por la adaptación a las condiciones favorables de los sistemas productivos (ambientes continuamente perturbados y alta cantidad de agua disponible) favoreciendo el desarrollo y producción de propágulos (distribución generalizada).

La composición florística hallada, es similar a las reportadas en investigaciones anteriores (Cardenal et al., 2016; Dotor et al., 2018), esto permite indicar que las especies encontradas hacen parte de la composición típica de malezas asociadas a suelos destinados a la producción zanahoria; se puede inferir que dichas comunidades de malezas persisten y están compuestas por las mismas especies. De acuerdo, con Poggio, (2012) indica que la composición florística de la comunidad de malezas está en función del clima, disponibilidad de nutrientes de agua, tipo de suelo, radiación solar y factores antrópicos. Cardenal et al. (2016) indican que el manejo cultural que el productor emplea en sus cultivos puede ser la causa de la selección de especies acompañantes.

Los índices de diversidad alfa ( $\alpha$ ) en estudios sobre poblaciones de malezas mide la cantidad de diversidad dentro de una comunidad definida en una 
zona; dentro de estos el índice de Shannon-Wiener se basa en la abundancia proporcional de cada especie y el índice de Simpson se basa en la probabilidad que dos individuos en una muestra sean de la misma especie (Booth et al., 2003). Estos son empleados de forma frecuente en estudios relacionados con la ecología de las malezas (Ramírez et al., 2015)

Se pudo observar que las áreas evaluadas en este estudio se diferenciaron por su diversidad. Según el índice de Shannon-Wiener, las zonas evaluadas presentaron una baja diversidad de especies con valores de 1, 44 en Ventaquemada y 1, 77 para Jenesano (Tabla IV). Según, Moreno, (2001) indican que valores menores a 2 representan una pobre diversidad en la comunidad evaluada. Por su parte, los índices de dominancia de Simpson presentaron valores mayores a 0,7 , esto indica que las áreas evaluadas tienen alta probabilidad de presentar especies dominantes (Tabla IV).

TABLA IV. Índice de diversidad Shannon Wiener e Índice de dominancia Simpson de la comunidad de malezas asociadas al cultivo de zanahoria en dos lotes dedicados a la producción de zanahoria de los municipios de Ventaquemada y Jenesano, Boyacá.

\begin{tabular}{|l|c|c|}
\hline Municipio & Índice de diversidad Shannon-Wiener & Índice dominancia Simpson \\
\hline Ventaquemada & 1,57 & 0,714 \\
\hline Jenesano & 1,77 & 0,743 \\
\hline
\end{tabular}

En términos generales, el coeficiente de dominancia de Simpson y el coeficiente de uniformidad indicaron que las comunidades estaban dominadas por pocas especies. Estos resultados se relacionan posiblemente con las prácticas realizadas control de las malezas en este sistema productivo, ya que cuando una comunidad de malezas es diversa, tiende a requerir tratamientos de control que afecten de forma diferencial la sensibilidad de las diferentes especies (Kuva et al., 2007).

Los índices de diversidad beta de Jaccard y Sorensen facilitan la comparación de áreas en términos de composición de las comunidades de malezas (Concenço et al., 2012). De acuerdo, con Booth et al. (2003) los valores deben interpretarse en una escala de 0 a 1 , donde 0 indica disimilitud total y 1 indica similitud absoluta. Los índices de similitud de Jaccard y Sorensen presentaron valores de 0,63 y 0,66 respectivamente. Esto indica similitud entre las comunidades de malezas en los municipios evaluados (Tabla $V$ ), esto posiblemente debido a la similiridad de prácticas de cultivo entre las zonas, como la poca rotación de cultivos y la intensificación de la siembra. Concenço et al. (2011) indican que zonas donde un cultivo se desarrolla de manera continua o sin rotación durante un largo período de tiempo, no habrá desconexión o disimilitud en las comunidades de malezas. Por su parte Ramírez et al. (2015) indican que el índice de Jaccard que varía de acuerdo con las condiciones climáticas y de manejo agronómico. 
TABLA V. Índice de diversidad Jaccard y Sorensen en el cultivo de zanahoria en dos lotes dedicados a la producción de zanahoria de los municipios de Ventaquemada y Jenesano, Boyacá.

\begin{tabular}{|l|c|}
\hline Índice & Ventaquemada-Jenesano \\
\hline Jaccard & 0,63 \\
\hline Sorensen & 0,66 \\
\hline
\end{tabular}

Los resultados del presente estudio concuerdan con los resultados encontrados por Cardenal et al. (2016) quienes al estudiar las composición del banco de malezas en el sistema productivo de zanahoria en el municipio de Villapinzon encontraron índices de Jaccard superiores al 0,6, esto debido a que las áreas estudiadas comparten el mismo sistema de producción, el tipo de vocación y el tipo de labranza convencional, siendo esto similar a los encontrado en los municipios analizados en este estudio.

\section{Conclusiones}

A través de la investigación se logró caracterizar que la comunidad de malezas en lotes destinados a la producción del cultivo de zanahoria en los municipios de Ventaquemada y Jenesano esta compuesta por 6 familias, siendo la de mayor importancia la familia Polygonaceae, ya que la especie $P$. nepalense y R. crispus se presentaron en las dos zonas evaluadas con mayores valores en el IVI. Los índices de diversidad permiten indicar que las zonas pueden presentar similaridad frente a las condiciones climáticas y de manejo del cultivo. Los resultados encontrados pueden servir de base y herramienta a los productores de zanahoria de las zonas evaluadas, para definir los planes de manejo de las malezas asociadas y así optimizar los rendimientos en este cultivo.

\section{Referencias}

Agronet. (2020). Estadisticas Agricolas. Área, producción, rendimiento y participación municipal en el departamento por cultivo. Estadisticas Agricolas. http:// www.agronet.gov.co/estadistica/Paginas/home.aspx?cod=1\#

Banda S., L., Fuentes, C. L., \& Chaves, B. (2012). Producción de semilla de cilantro (Coriandrum sativum L.) bajo la incidencia de malezas y Alternaria Nees. Revista Colombiana de Ciencias Hortícolas, 5(2), 251-262. https://doi.org/10.17584/ rcch.2011v5i2.1274

Bastidas, J., \& López, G. (1972). Caracteres cuantitativos y reconocimientos de malezas en praderas artificiales de clima frio del altiplano de Pasto. Rev. Ciencias Agrícolas, 4(2), 83-97.

Biffe, D. F., Constantin, J., \& Oliveira Junior, R. S. de. (2018). Interferência das plantas daninhas nas plantas cultivadas. In Hortaliças-fruto-EDUEM, 339-355.. https:// doi.org/10.7476/9786586383010.0012 
Booth, B. D., Murphy, S. D., \& Swanton, C. J. (2003). Weed Ecology in Natural and Agricultural Systems. In CABI Publishing. 313 p.

Cabrera, D. C., Chaila, S., Sobrero, M. T., \& Varela, A. E. (2019). Phytosociological survey of sugarcane crop weeds in different agroecological areas in TucumÁn Province, Argentina. Planta Daninha, 37, 31-38. https://doi.org/10.1590/ S0100-83582019370100027

Cardenal Rubio, C. Z., Torres Hernández, D., Dotor Robayo, M. Y., \& Morillo Coronado, A. C. (2016). Characterization of the active seed bank in carrot crops in the municipallity of Villa Pinzón (Cundinamarca). Rev. U.D.C.A Act. \& Div. Cient., 19(2), 297-306.

Carvalho, L. B., \& Guzzo, C. D. (2008). Increased beet density in weed management. Planta Daninha, 26(1), 73-82. https://doi.org/10.1590/ S0100-83582008000100008

Carvalho, L. B., Pitelli, R. A., Cecílio Filho, A. B., Bianco, S., \& Guzzo, C. D. (2008). Interferência e estudo fitossociológico da comunidade infestante em beterraba de semeadura direta. Planta Daninha, 26(2), 291-299. https://doi.org/10.1590/ s0100-83582008000200005

Coelho, M., Bianco, S., \& Carvalho, L. (2009). Interferência de plantas daninhas na cultura da cenoura (Daucus carota). Planta Daninha, 27(spe), 913-920. https:// doi.org/10.1590/s0100-83582009000500004

Concenço, G., Silva, C. J., Staut, L. A., Pontes, C. S., Laurindo, L. C. A. S., \& Souza, N. C. D. S. (2012). Weeds occurrence in areas submitted to distinct winter crops. Planta Daninha, 30(4), 747-755. https://doi.org/10.1590/ S0100-83582012000400008

Concenço, G, Salton, J. ., Secretti, M. ., Mendes, P. ., Brevilieri, R. ., \& Galon, L. (2011). Effect of long-term agricultural management systems on occurrence and composition of weed species. Planta Daninha, 29(3), 515-522. https://doi. org/10.1590/s0100-83582011000300005

Concenço, Germani, Farias, P. M. de, Quintero, N. F. A., Schreiber, F., Galon, L., Tomazi, M., Moisinho, I. S., Coradini, M. C., Ceolin, W. C., \& Andres, A. (2017). Phytosociological Surveys in Weed Science: Old Concept, New Approach. Plant Ecology - Traditional Approaches to Recent Trends. https://doi.org/10.5772/ intechopen.69083

Dotor R., M. Y., González Mendoza, L. A., \& Morillo C., A. C. (2018). Período crítico de competencia de la Zanahoria (Daucus carota L.) y malezas asociadas al cultivo. Revista de Ciencias Agrícolas, 35(1), 5. https://doi.org/10.22267/ rcia.183501.78

Fuentes, C., Eraso, E., Sequeda, O., \& Piedrahita, W. (2011). Flora arvense del antiplano Cundiboyacense de Colombia (B. CropScience \& Universidad Nacional de Colombia (eds.). 350 p.

Gámez, A., Rojas, L., Jaramillo, M., Cruz, E., Hoyos, V., Cepeda, J., \& Plaza, G. (2018). Guía Ilustrada De Plantas Arvenses Del Centro Agropecuario Marengo (Cam). 382 p.

Kuva, M. A., Pitelli, R. A., Salgado, T. P., \& Alves, P. L. C. A. (2007). Fitossociologia de comunidades de plantas daninhas em agroecossistema cana-crua. Planta Daninha, 25(3), 501-511. https://doi.org/10.1590/S0100-83582007000300009 
López, A. J. G., Hernández, M., \& Díaz, R. (2012). Caracterización de la flora arvense asociada a un cultivo de maíz bajo riego para producción de jojotos. Agronomía Trop., 61 (2), 133-139.

Moreno, C. (2001). Métodos para medir la biodiversidad, edición electrónica. (M\&TManual, Issue January 2001). 84p.

Patkowska, E. (2021). Cover crops and soil-borne fungi dangerous towards the cultivation of daucus carota L. Acta Scientiarum Polonorum, Hortorum Cultus, 20(2), 3-12. https://doi.org/10.24326/asphc.2021.2.1

Pitelli, R. A. (1987). COMPETIÇÃO E CONTROLE DAS PLANTAS DANINHAS EM ÁREAS AGRíCOLAS. Série Técnica IPEF, Piracicaba, 4(12), 1-24.

Plaza, G. A., \& Pedraza, M. (2007). Reconocimiento y caracterización ecológica de la flora arvense asociada al cultivo de uchuva. Agronomia Colombiana, 25(2), 306-313.

Poggio, S. L. (2012). Cambios florísticos en comunidades de malezas: Un marco conceptual basado en reglas de ensamblaje. Ecologia Austral, 22(2), 150-158.

Poleshi Chaitra, A., Manikanta, D. S., \& Cholin, S. (2018). Understanding the genetic variability, heritability and association pattern for the characters related to reproductive phase of carrots (Daucus carota L.) in tropical region. Journal of Applied Horticulture, 20(3), 225-232. https://doi.org/10.37855/JAH.2018. V20103.39

Prates, C. J. N., Viana, A. E. S., Cardoso, A. D., São José, A. R., Viana, B. A. R., \& Dutra, F. V. (2019). Weed Phytosociology in Cassava Cultivation in Two Periods in Southwestern Bahia, Brazil. Planta Daninha, 37(019184596), 1-12. https:// doi.org/10.1590/s0100-83582019370100107

Quintero-Pertuz, I., Carbonó-Delahoz, E., Hoyos, V., Jarma-Orozco, A., \& Plaza, G. (2021). Phytosociology of weeds in banana plantations in the department of Magdalena, Colombia. Caldasia, 43(1), 80-93. https://doi.org/10.15446/caldasia.v43n 1.83554

Ramírez, J., Hoyos, V., \& Plaza, G. (2015). Fitosociología de malezas asociadas al cultivo de arroz en el departamento del Tolima, Colombia. Agronomia Colombiana, 33(1), 64-73. https://doi.org/10.15446/agron.colomb.v33n1.46747

Reginaldo, L. T. R. T., Lins, H. A., Sousa, M. D. F., Teófilo, T. M. D. S., Mendonça, V., \& Silva, D. V. (2021). Weed interference in carrot yield in two localized irrigation systems. Revista Caatinga, 34(1), 119-131. https://doi. org/10.1590/1983-21252021v34n113rc

Resende, G. M., Yuri, J. E., Costa, N. D., \& Mota, J. H. (2016). Desempenho de cultivares de cenoura em sistema orgânico de cultivo em condições de temperaturas elevadas. Horticultura Brasileira, 34(1), 121-125. https://doi.org/10.1590/ S0102-053620160000100018

Schmid, C., Sharma, S., Stark, T. D., Günzkofer, D., Hofmann, T. F., Ulrich, D., Dunemann, F., Nothnagel, T., \& Dawid, C. (2021). Influence of the abiotic stress conditions, waterlogging and drought, on the bitter sensometabolome as well as agronomical traits of six genotypes of daucus carota. Foods, 10(7). https://doi. org/10.3390/foods 10071607

Vera Ojeda, P. A., \& Enciso Garay, C. R. (2017). Critical period of weed interference in carrot crop. Investigación Agraria, 19(2), 77-85. https://doi.org/10.18004/ investig.agrar.2017.diciembre.77-85 